\title{
Pro-inflammatory pattern of lgG1 Fc glycosylation in multiple sclerosis cerebrospinal fluid
}

\author{
Manfred Wuhrer ${ }^{1,2,3}$, Maurice H. J. Selman' ${ }^{1}$, Liam A. McDonnell', Tania Kümpfel ${ }^{4}$, Tobias Derfuss ${ }^{5}$, \\ Mohsen Khademi ${ }^{6}$, Tomas Olsson ${ }^{6}$, Reinhard Hohlfeld ${ }^{4,7}$, Edgar Meinl ${ }^{4}$ and Markus Krumbholz ${ }^{4,8^{*}}$
}

\begin{abstract}
Background: Immunoglobulin G (IgG) effector functions are regulated by the composition of glycans attached to a conserved N-glycosylation site in the Fc part. Intrathecal production of $\operatorname{lgG}$, especially lgG1, is a hallmark of multiple sclerosis (MS), but nothing is known about lgG Fc glycosylation in MS and in cerebrospinal fluid (CSF) in general.

Methods: We applied mass spectrometry of tryptic Fc glycopeptides to analyze lgG Fc glycosylation (sialylation, galactosylation, fucosylation, and bisecting N-acetylglucosamine (GlcNAc)) in 48 paired CSF and serum samples from adult patients with MS or a first demyelinating event highly suggestive of MS (designated as MS cases), and from healthy volunteers and patients with other non-inflammatory diseases (control group). $p$ values were adjusted for multiple testing.

Results: Our experiments revealed four main results. First, lgG1 glycosylation patterns were different in CSF vs. serum, in the MS group and even in control donors without intrathecal lgG synthesis. Second, in MS patients vs. controls, IgG1 glycosylation patterns were altered in CSF, but not in serum. Specifically, in CSF from the MS group, bisecting GIcNAc were elevated, and afucosylation and galactosylation were reduced. Elevated bisecting GlcNAc and reduced galactosylation are known to enhance $\mathrm{lgG}$ effector functions. Third, hypothesis-free regression analysis revealed that alterations of afucosylation and bisecting GICNAC in CSF from MS cases peaked 2-3 months after the last relapse. Fourth, CSF IgG1 glycosylation correlated with the degree of intrathecal lgG synthesis and CSF cell count.
\end{abstract}

Conclusions: The CNS compartment as well as the inflammatory milieu in MS affect IgG1 Fc glycosylation. In MS, the CSF IgG1 glycosylation has features that enhance Fc effector functions.

Keywords: Multiple sclerosis, Cerebrospinal fluid, Immunoglobulin G, Glycosylation

\section{Background}

Intrathecal immunoglobulin G (IgG) production is a hallmark of multiple sclerosis (MS) [1, 2]. There is strong evidence for IgG-mediated pathomechanisms at least in a subset of patients with MS, although the precise autoantigen remains to be identified for most patients [2-7]. IgG effector mechanisms via complement

\footnotetext{
* Correspondence: markus.krumbholz@uni-tuebingen.de

${ }^{4}$ Institute of Clinical Neuroimmunology, Biomedical Center (BMC) and University Hospital, Campus Martinsried-Grosshadern, LMU Munich, Munich, Germany

${ }^{8}$ Department of Neurology and Stroke, and Hertie Institute for Clinical Brain Research, University of Tübingen, Tübingen, Germany

Full list of author information is available at the end of the article
}

and $\mathrm{Fc}$ gamma receptors $(\mathrm{Fc} \gamma \mathrm{Rs})$ are regulated by the glycan composition at a conserved $\mathrm{N}$-glycosylation site (asparagine 297) in the $\mathrm{Fc} \mathrm{CH}_{2}$ domain of the heavy chain $[8,9]$.

The functional in vivo relevance of different IgG Fc glycosylation patterns has been shown in animal models of systemic autoimmune diseases [10-14]. The presence or absence of certain sugar residues (Fig. 1) has been linked to pro- or anti-inflammatory properties: terminal sialylation confers anti-inflammatory properties, and the sialylated fraction of therapeutic intravenous immunoglobulins (IVIG) was suggested to contribute to the therapeutic effect of IVIG $[15,16]$,

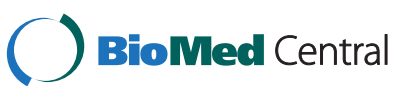

(c) 2015 Wuhrer et al. Open Access This article is distributed under the terms of the Creative Commons Attribution 4.0 International License (http://creativecommons.org/licenses/by/4.0/), which permits unrestricted use, distribution, and reproduction in any medium, provided you give appropriate credit to the original author(s) and the source, provide a link to the Creative Commons license, and indicate if changes were made. The Creative Commons Public Domain Dedication waiver (http://creativecommons.org/publicdomain/zero/1.0/) applies to the data made available in this article, unless otherwise stated. 


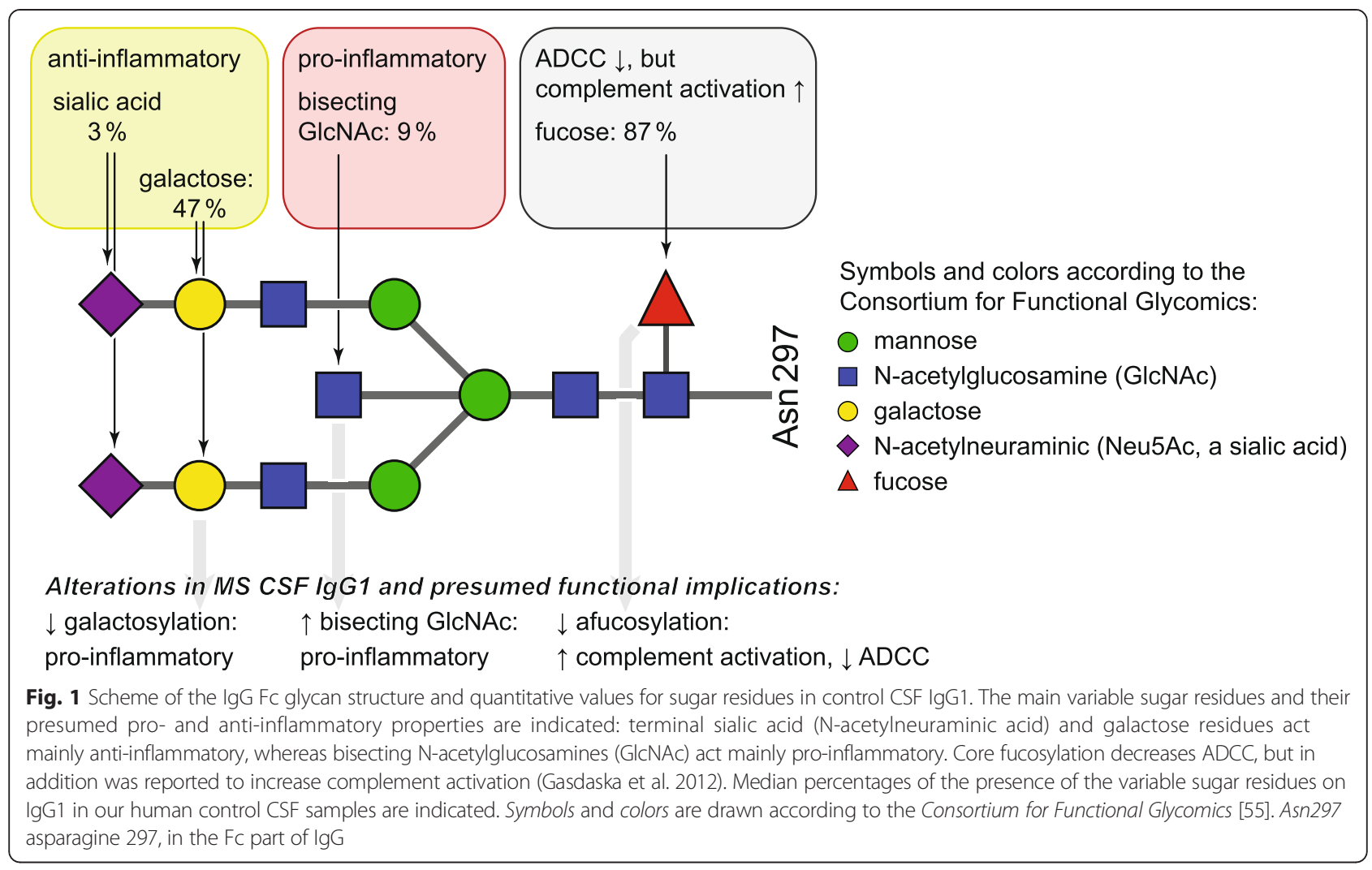

although the exact downstream mechanisms may differ between species [12, 17, 18]. Likewise, galactosylation confers anti-inflammatory properties, since decreased galactosylation of IgG resulted in increased pathogenicity in autoantibody-mediated murine models of autoimmune diseases [10, 11, 19]. In contrast, bisecting $\mathrm{N}$-acetylglucosamines (GlcNAc) are pro-inflammatory, e.g., by enhancing antibody-dependent cellular cytotoxicity (ADCC) $[8,20,21]$. Removal of core fucose residues selectively enhances the affinity of IgG for human activating FcrRIIIa, while the binding to all other activating Fc gamma receptors is not affected [8], but complement activation seems to be reduced [22]. Removal of pro-inflammatory glycans by glycosidases such as PNGase F or EndoS abrogates IgG pathogenicity in animal models $[13,14]$.

Glycosylation of IgG in blood is altered in human autoimmune diseases: in serum from patients with systemic autoimmune diseases such as rheumatoid arthritis, Lambert-Eaton myasthenic syndrome, and Guillain-Barré-Syndrome [23-27], IgG Fc glycosylation is altered toward a more pro-inflammatory pattern. A proinflammatory glycosylation pattern precedes clinical disease onset of human rheumatoid arthritis [28]. Importantly, the pathogenic impact of IgG Fc glycosylation has been demonstrated mechanistically with human IgG: anti-aquaporin-4 autoantibodies from patients with neuromyelitis optica (NMO) induce NMO-like lesions in mouse transfer models $[29,30]$, and this pathogenic effect of NMO-Ig is abrogated by deglycosylation before transfer [31].

The translation into a therapeutic in vivo approach has been pioneered using the IgG-specific endoglycosidase EndoS [32]: in vivo injection of EndoS-diminished $\mathrm{MOG}_{35-55}$ induced experimental autoimmune encephalomyelitis as a model of MS [33], as well as anti-GBM and ANCA mediated glomerulonephritis in rodent models [34, 35], and SLE-like disease in BXSB mice [14]. The regulation of IgG effector functions by glycosylation is further utilized by IgG glycol-engineering of therapeutic monoclonal antibodies according to their desired properties [36, 37].

We present the first analysis of IgG glycosylation in the cerebrospinal fluid (CSF) and in MS. We applied mass spectrometry to determine IgG Fc glycosylation in paired CSF/serum samples and addressed the following questions: (1) Does IgG1 glycosylation differ between CSF and serum in the absence of inflammation? (2) Is multiple sclerosis associated with an altered pattern of IgG1 glycosylation in CSF or serum? (3) Is the pattern of CSF IgG1 glycosylation associated with time since last relapse and markers of inflammation in the CSF such as cell count and intrathecal IgG synthesis? 


\section{Methods}

\section{Patients and healthy volunteers}

We evaluated 48 paired serum and CSF samples. Thereof, 27 were from patients with definite MS diagnosed according to the $2005 \mathrm{McDonald}$ criteria or a first demyelinating event ("clinically isolated syndrome", CIS) highly suggestive of incipient MS (MS subgroups, MSCIS $(n=10)$, relapsing-remitting MS (MS-RR, $n=12$ ), secondary progressive MS (MS-SP, $n=2$ ), primary progressive MS (MS-PP, $n=3$ ); all summarized as MS group). Even though the 2010 McDonald criteria allow for an earlier diagnosis, we adhered to the 2005 criteria since original MRI images were not available for all MS cases for reassessment. Since we were especially interested in CSF IgG, patients were selected for a high intrathecal IgG production according to the Reiber formula [38] (intrathecal fraction of $\operatorname{IgG}\left(\mathrm{IF}_{\mathrm{IgG}}\right)$ mean $36 \%$, median $44 \%$, interquartile range (IQR) 9 to $52 \%$ ). From these 27 MS cases, 18 had a recent ( $<90$ days) clinical relapse (median interval 19 days, IQR 8-42 days). Nine patients had received steroids within 90 days, and one patient natalizumab. Two patients had concomitant uveitis, one of whom received methotrexate. We analyzed $21 \mathrm{CSF} /$ serum sample pairs from control donors, including 5 healthy control volunteers $(\mathrm{HC})$ and 16 patients with other, non-inflammatory neurological disease (OND, e.g., tension headache, migraine, pseudotumor cerebri, normal tension hydrocephalus, cerebral ischemia, diabetic neuropathy, and panic disorder). From the donors described, CSF and serum was analyzed, but some mass spectrometric peaks could not be quantified, so that $2 \%$ of data points were missing. From four additional donors $(2 \times \mathrm{MS}, 2 \times \mathrm{OND})$, serum profiles could be obtained.

Further characteristics of the study cohort are shown in Table 1. Subgroup analysis did not reveal significant differences in glycosylation between the subgroups of the control group ( $\mathrm{HC}$ and $\mathrm{OND}$ ) or the MS group (CIS, MS-RR, and MS-CP patients). A change of IgG glycosylation with age has been described in serum [39, 40],

Table 1 Study cohorts: number of samples and clinical characteristics

\begin{tabular}{lll}
\hline & MS group & Controls \\
\hline Number & 27 & 21 \\
Women (percentage) & $16(59 \%)$ & $11(52 \%)$ \\
Age in years $^{\mathrm{a}}$ & $36(28-43)$ & $33(27-48)$ \\
Disease duration $^{\mathrm{a}}$ & 1.0 year (36 days-6.9 years) & n.a. \\
EDSS $^{\mathrm{a}}$ & $2.5(2,3)$ & n.a. \\
Albumin quotient $^{\left(Q_{\mathrm{alb}}\right)^{\mathrm{a}}}$ & $5.4(4.1-6.8])$ & $4.6(3.2-7.6)$ \\
${\text { CSF cell count } /\left.\mu\right|^{\mathrm{a}}}$ & $13(3.8-20.2)$ & $2(1-2.3)$ \\
\hline
\end{tabular}

data are given as median (interquartile range) EDSS expanded disability status scale, n.a., not applicable and we noted such correlations of IgG glycosylation with age also in CSF IgG. Importantly, however, the similar median age (Table. 1), similar age distribution, and further regression analysis (data not shown) excluded age as a relevant bias for our study.

\section{Ethics, consent, and permissions}

Informed consent was obtained from all patients and healthy volunteers according to local ethics committee regulations (Medical Faculty of the University of Munich, project 159/03; University Erlangen-Nuremburg, project 4203; Karolinska Institute, project Stop MS II, 2009/2107-31/2).

\section{Basic CSF and serum analysis}

CSF cell counts, IgG, and albumin concentrations in CSF and serum were analyzed at each center separately with standard methods as part of the routine patient workup using highly standardized and accurate methods approved for diagnostic use. To ensure accuracy, regular quality controls including round robin tests are performed as applicable. The IgG quotient $\left(Q_{\mathrm{IgG}}\right)$ is defined as the ratio of the concentrations of IgG in CSF divided by IgG in serum. As a more elaborate method to quantify the fraction of IgG that is produced intrathecally $\left(\mathrm{IF}_{\mathrm{IgG}}\right)$, we applied this formula $\left(\mathrm{IF}_{\mathrm{IgG}}=1-\frac{Q_{\lim }(\mathrm{IgG})}{Q_{\mathrm{IgG}}}\right.$, with $\left.Q_{\lim }(\operatorname{IgG})=0.93 \sqrt{Q_{\mathrm{alb}}{ }^{2}+6 \times 10^{-6}}-1.7 \times 10^{-3}\right)$, which is based on the work of Reiber and Peter [38].

\section{Mass spectrometry}

Serum and CSF were collected in all sites following the same protocol. CSF and serum samples were centrifuged immediately. Aliquots of cell-free CSF and serum supernatant were stored at $-80{ }^{\circ} \mathrm{C}$ immediately and shipped later on dry ice. Mass spectrometry for the glycosylation profiles of Fc-derived glycopeptides was performed in the same lab and same experimental setup following a recently established protocol [41]. Briefly, IgG was purified from serum or CSF using protein A affinity capturing in the 96-well plate format. Purified IgG was subjected to tryptic digestion, and resulting glycopeptides were desalted by reverse phase-solid phase extraction. Glycopeptides were analyzed using a 9.4 T Apex Q matrix-assisted laser desorption/ionization Fourier transform ion cyclotron resonance mass spectrometer (Bruker Daltonics, Bremen, Germany). An example of the obtained Fc glycosylation profiles of paired serum and CSF samples is shown in Additional file 1: Figure S1. Detected IgG Fc glycopeptide signals were integrated. For both the IgG1 and IgG2 subclass, the sum of signal was set to $100 \%$. From these data, the abundance of IgG1 and IgG2 Fc N-glycan structural features was calculated, including galactosylation, bisecting $\mathrm{N}$-acetylglucosamine 
(GlcNAc), sialylation, and core fucosylation. Fucosylation was only assessed for IgG1 and not for IgG2 as several fucosylated IgG2 glycoforms could not be determined due to overlay with IgG4 glycopeptides. Data is presented as percentage for each glycosylation feature: e.g., 47 \% galactosylation indicates that $47 \%$ of canonical galactose residues according to the scheme in Fig. 1 were actually present. We always plotted the proportion of the less common form (e.g., sialylation, but afucosylation $(\sim 13 \%)$ instead of fucosylation $(87 \%))$.

\section{Statistics and normalization of IgG glycosylation in CSF and serum}

Nonparametric tests were used throughout the manuscript. All tests were two-sided unless indicated otherwise. All statistical tests, adjustments for multiple testing, and plotting of data were performed in R [42].

Comparisons between two groups were calculated by Mann-Whitney $U$ test for unpaired samples and by Wilcoxon-signed rank test for paired samples. All $p$ values from group comparisons were adjusted for multiple testing $\left(p_{\text {adj }}\right)$ across all comparisons (sugar residues and IgG subclasses) as family-wise error rate (Bonferroni correction). When depicting glycosylation as absolute values in CSF and serum separately (=2 compartments), this was also taken into account, resulting in a higher correcting factor for absolute CSF and serum values (4 sugars $\times 2$ IgG subclasses $\times 2$ compartments $=16$ ), compared to CSF/serum ratios (4 sugars $\times 2$ IgG subclasses $\times$ only 1 ratio of both compartments $=8$ ). Boxplots were plotted with default whiskers from $\mathrm{R}$ (range up to $1.5 \times \mathrm{IQR}$ below/above first/third quartile).

Correlations between two parameters were calculated by Spearman's method. $p$ values were adjusted for multiple testing across all possible comparisons of glycoforms and (para-)clinical observations including galactosylation, sialylation, bisecting GlcNAc, afucosylation, age at LP, disease duration, time from last relapse, CSF cell count, $Q_{\text {alb }}, Q_{\mathrm{IgG}}, \mathrm{IF}_{\mathrm{IgG}}, \mathrm{EDSS}$ ) as false discovery rates [43] separately for each donor group. Consistent with nonparametric correlation statistics, trendlines were computed as robust locally weighted regression and smoothing scatterplot (LOWESS) lines [44]. When plotting categorical data on the $x$-axis, data points were jittered horizontally within each category to avoid them obscuring each other. Colors were chosen for best contrast also for colorblind people. We noted that MS cases differed from controls regarding IgG glycosylation in CSF, but not in serum, and normalized CSF glycosylation data as CSF/serum ratio. Using $\mathrm{CSF} /$ serum ratios instead of absolute CSF glycosylation values reduced noise (defined as geometric mean of the interquartile ranges of both groups, divided by the difference of medians of both groups) by about $60 \%$, but did not affect the direction of differences in between the groups (Figs. 2 and 4). Therefore, the CSF/serum ratio for IgG glycosylation was more sensitive and stable, and was used for comparison of groups.

Principal component analysis was performed by the function princomp from the stats package in R. Principal component analysis axis scales for score and loading were plotted according to the biplot standard.

\section{Results}

CSF vs. serum IgG1 glycosylation differs, both in controls and in MS cases

We quantified IgG1 Fc glycosylation in CSF and serum. A scheme of the attached oligosaccharides, along with frequencies of the respective variable sugar residues in control CSF IgG1, is shown in Fig. 1. A representative mass spectrum is shown in Additional file 1: Figure S1.

We noted differences between CSF and serum IgG1 glycosylation (Fig. 2). In CSF from MS cases, glycans containing bisecting GlcNAc were increased, whereas galactosylated and sialylated species were reduced. Unexpectedly, differences between CSF and serum were detected also in the control group without intrathecal IgG production; in control CSF, galactosylation was reduced as well, while sialylation and bisecting GlcNAc were only slightly (n.s.) shifted into the same direction as in MS CSF. Notably, we observed an increase of afucosylated IgG1 in the CSF of control donors compared to serum that was lacking in the MS cases. Similar results were obtained for IgG2 (Additional file 2: Figure S2).

While the absolute level of glycosylation differed between CSF and serum, we observed a positive correlation between identical IgG1 glycoforms in serum vs. CSF (Fig. 3). This correlation was much higher for the group of control donors (median $\varrho 0.91, p_{\text {adj }}<0.005$ for each glycosylation feature) than for the MS group (median $\varrho 0.48, p_{\text {adj }}<0.05$ only for bisecting GlcNAc), consistent with an intrathecal IgG production in addition to plasma-derived IgG in the CSF from MS cases. Correlations between serum and CSF IgG2 were less pronounced as compared to IgG1 but present especially for IgG2 galactosylation both in MS and controls ( $\varrho 0.95$ and 0.94 , $p_{\text {adj }}<0.00001$ for both).

\section{IgG1 glycosylation is altered in CSF from MS cases vs. controls}

Comparing MS cases with controls, IgG1 Fc glycosylation was altered in CSF, but not in serum (Fig. 2). CSF/serum ratios were more sensitive and stable than the absolute CSF values for comparison of groups (see Methods) and are used in the following. Bisecting GlcNAc of IgG1 was increased in CSF from MS cases. This was evident by the CSF/serum ratio $\left(1.3 \times, p=0.0005, p_{\text {adj }}=0.004\right.$; Fig. 4a) and by the absolute CSF values $(1.3 \times, p=0.0021$, $p_{\text {adj }}=0.034$; Fig. 2). IgG1 galactosylation was slightly but 


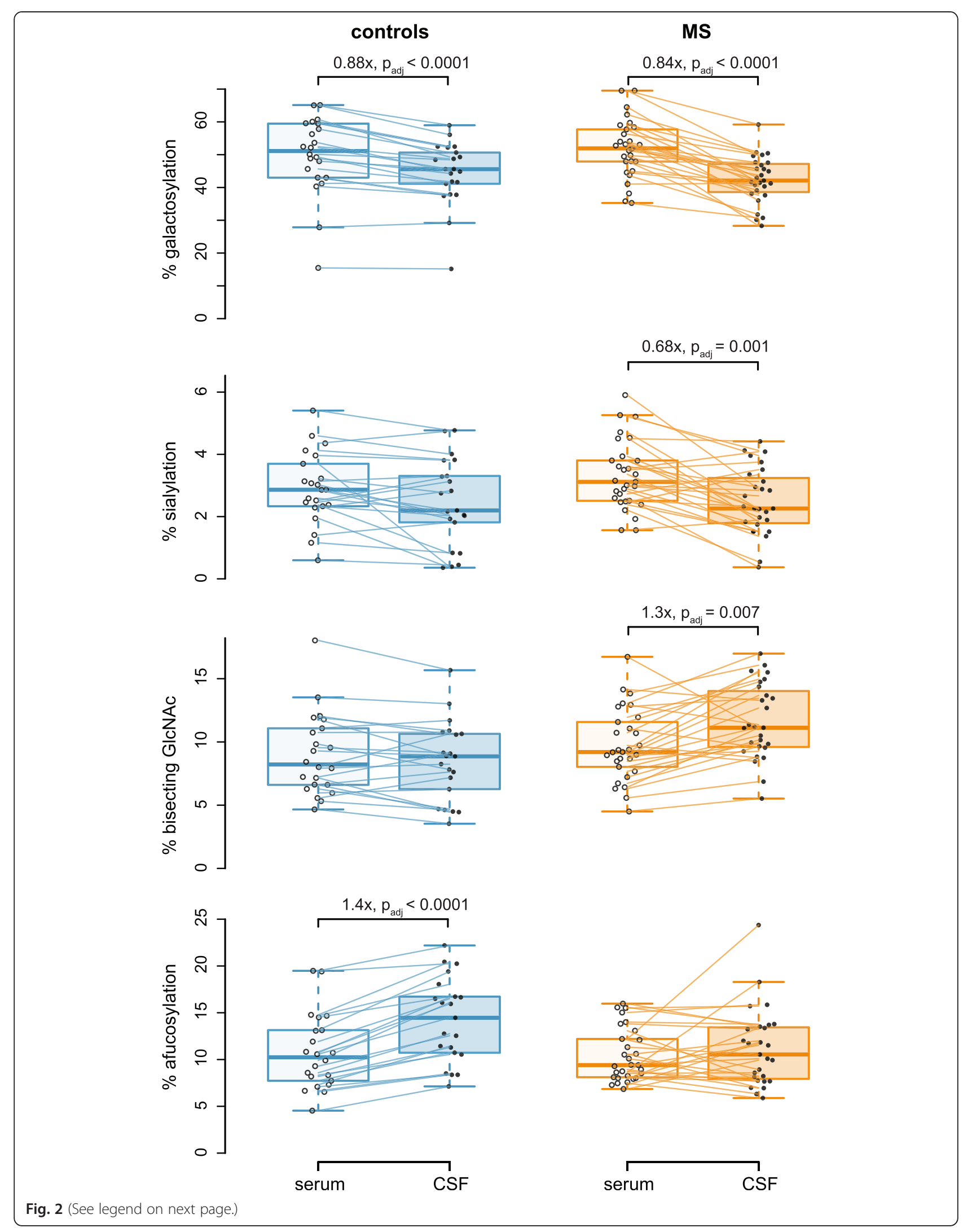


(See figure on previous page.)

Fig. 2 lgG1 glycosylation differs in CSF vs. serum. IgG1 glycosylation was quantified by mass spectrometry in paired CSF/serum samples from 48 donors. Individual data points are shown. Lines indicate corresponding CSF/serum pairs, but do not necessarily end directly at the horizontally jittered data points to preserve angles of the connecting lines. Significance was determined using Wilcoxon-signed rank test for paired samples, followed by Bonferroni correction for multiple testing $\left(p_{\text {add }}\right)$. Factors above diagrams indicate fold-changes (medians of paired CSF/serum ratios). Please note that we always show the less abundant glycoform, i.e., afucosylated lgG in the lowest panel

significantly decreased in MS CSF $(0.96 \times, p=0.0026$, $p_{\text {adj }}=0.021 ;$ Fig. $\left.4 \mathrm{~b}\right)$. The decrease of IgG1 sialylation (N-acetylneuraminic acid) did not reach statistical significance (Fig. 4c, $p=0.08$ ). The CSF/serum ratio of afucosylated IgG1 glycoforms was decreased in MS compared to control donors $\left(0.71 \times, p=0.0008, p_{\text {adj }}=0.007\right.$; Fig. $\left.4 d\right)$.

Principal component analysis revealed that all four glycosylation features together classified CSF samples better as a MS or control sample than each single glycosylation feature alone, resulting in a good group separation (Fig. 4e). In fact, classification quality according to post-hoc defined criteria (ellipses in Fig. 4e) in our cohort was similar to CSF cell count and intrathecal IgG fraction (principal component analysis; sensitivity $74 \%$, specificity $100 \%$; CSF cell count $>5$ cells; sensitivity $65 \%$ (specificity $100 \%$, a priori definition of control samples); positive intrathecal IgG fraction; sensitivity $74 \%$ (specificity $100 \%$, a priori definition of control samples)). Taken together, IgG1 glycofeatures separated our MS patients from controls as good as CSF cell count or intrathecal IgG production, albeit not as sensitive as oligoclonal bands.

Next, we analyzed if MS-associated changes of IgG1 glycosylation were more pronounced in a time period related to a relapse. We sought for a hypothesis-free definition for such a period and computed LOWESS regression lines (red curve in Fig. 5). The time between the crosses of their peak with the median of all samples (horizontal line) was designated as the peak period for glycosylation changes. Alterations in afucosylation and bisecting GlcNAc culminated 2-3 months after the last relapse (Fig. 5, left panel) and were significantly more pronounced in samples from within this peak period, compared with samples from outside this period or with control samples (Fig. 5, right panel). Since this analysis was an unplanned subgroup analysis, we did not plan correction for multiple testing, but Bonferroni correction for all four glycosylation features would have left significant results for afucosylation (peak vs. both nonpeak and controls) and bisecting GlcNAc (peak vs. controls). In contrast, we could not detect a substantial influence of the time since last relapse on galactosylation and sialylation. Neither could we detect an effect of therapy including steroids on glycosylation, but this study was not powered to detect such effects.

The pattern of alterations between MS CSF and control CSF described here was different from that between control CSF and control serum described in Fig. 3 (summarized in Table 2). In contrast to the differences observed for IgG1 glycosylation in CSF from MS cases vs. controls, such differences for IgG2 glycosylation were present only as a trend or not at all (Additional file 3: Figure S3).

\section{Correlation of IgG glycosylation with CSF cell count and intrathecal lgG fraction}

MS-related changes in Fc glycosylation were more pronounced in those patients with higher cell counts (Fig. 6a). The correlation with CSF cell counts was strongest for afucosylated IgG1 $\left(\varrho-0.83, p<0.00001, p_{\text {adj }}<0.00001\right)$ and present as a trend after correction for multiple testing for galactosylation and bisecting GlcNAc.

We further noted a negative correlation of afucosylated IgG1 with both $Q_{\operatorname{IgG}}$ and the intrathecally produced IgG fraction $\left(\mathrm{IF}_{\mathrm{IgG}}\right)$ according to the Reiber formula [38] (Fig. 6b; $Q_{\mathrm{IgG}} ; \varrho=-0.65, p<0.001, p_{\text {adj }}=0.004 ; \mathrm{IF}_{\mathrm{IgG}}$; $\left.\varrho=-0.61, p<0.001, p_{\text {adj }}=0.012\right)$. This is consistent with an intrathecal production of less afucosylated IgG, as might be inferred also from the group comparisons (Fig. 4). For IgG1 galactosylation and bisecting GlcNAc, similar trends for an association with $Q_{\mathrm{IgG}}$ and $\mathrm{IF}_{\mathrm{IgG}}$ were present and paralleled the observed group differences. Since there is no intrathecal IgG production in control donors, this was only analyzed within the MS group.

There was no significant correlation of any glycosylation feature with disease duration or EDSS, but the study was not powered to detect associations with these clinical parameters.

\section{Associations between different glycoforms}

When assessing different IgG glycosylation features within the same compartment (CSF or serum) and same group (MS cases or controls), we noted that by far the strongest positive correlation existed between sialylation and galactosylation (for control and MS donors, for CSF and serum IgG1; $\varrho=0.67$ to $0.93, p_{\text {adj }}<0.01$ for each; Additional file 4: Figure S4A). In contrast, there was no association for all other combinations of glycoforms (Additional file 4: Figure S4B, exemplified for galactosylation vs. bisecting GlcNAc and afucosylation).

\section{lgG2 vs. IgG1 glycosylation}

The glycosylation pattern was significantly different for IgG2 vs. IgG1 in both serum and CSF from controls and 
A) correlation of CSF vs. serum IgG1 glycosylation

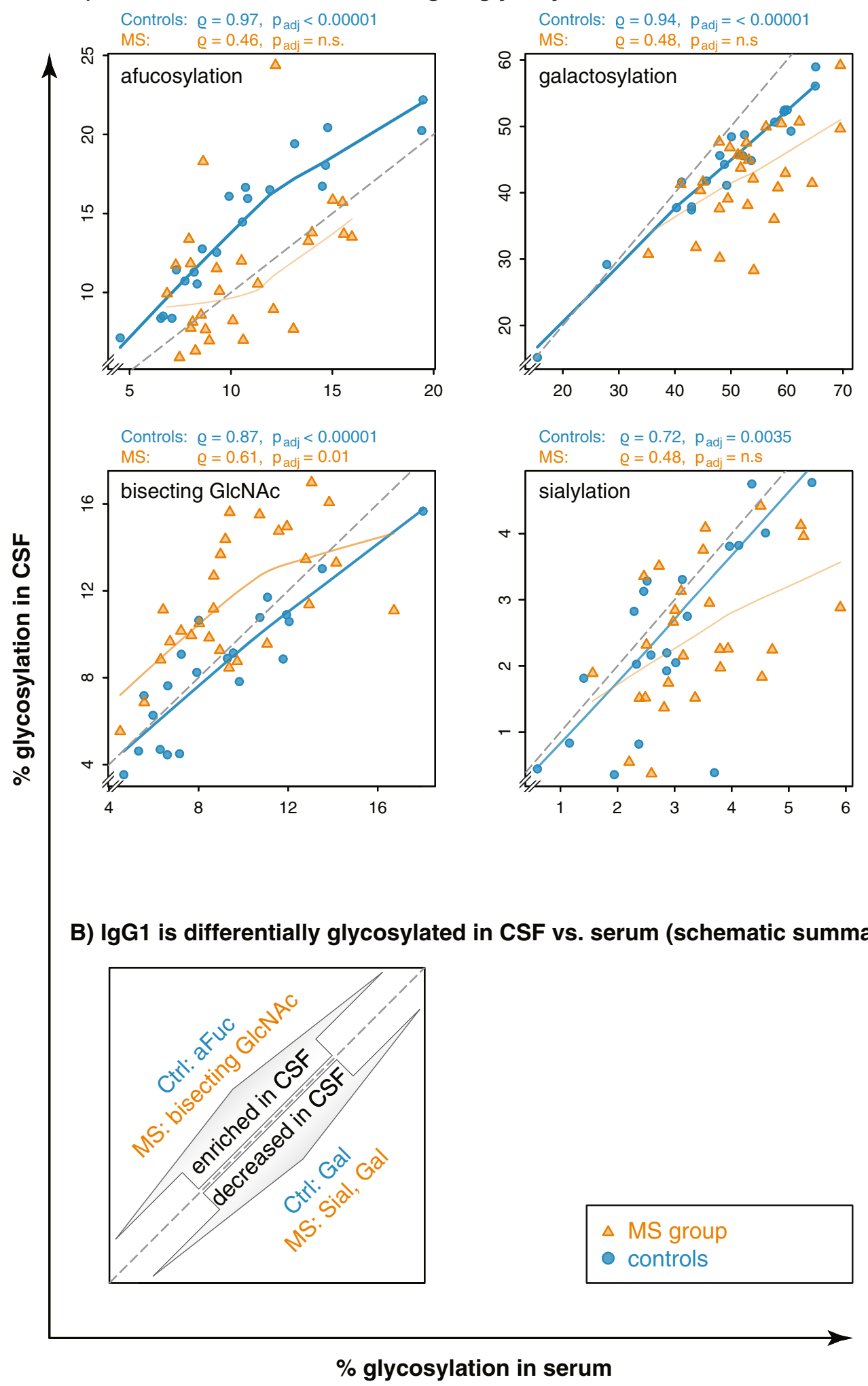

Fig. 3 (See legend on next page.) 
(See figure on previous page.)

Fig. 3 Strong correlation of sugar residues in serum vs. CSF in controls, but much less in the MS group. a lgG1 afucosylation, bisecting GlcNAc, galactosylation, and sialylation strongly correlate in CSF vs. serum from control donors (blue circles) which do not have intrathecal lgG synthesis. In contrast, the correlation is much weaker within the MS group (orange triangles), consistent with additional intrathecal lgG synthesis. $\varrho$ and $p$ values (Spearman's method, adjusted for multiple testing) are given for each sugar residue. Trendlines represent LOWESS lines (Cleveland 1979) and indicate the strength of association (Spearman's $\varrho$ ) by their opacity and thickness. Dashed gray lines represent the angle bisectors, indicating where CSF and serum glycosylation would be equal. Thus, each data point that is located left/above this line indicates a sample pair where the respective feature is overrepresented in CSF, whereas each data point right/below this line indicates a sample pair where the respective feature is overrepresented in the serum. This is summarized schematically in $\mathbf{b}$, showing that lgG1 is differentially glycosylated in CSF vs. serum. For selection of significant differences between CSF and serum, paired Wilcoxon tests were used with Bonferroni correction for multiple testing (see also Fig. 2). In MS patients (orange), bisecting GICNAc were enriched in CSF, whereas CSF IgG1 was less sialylated and galactosylated compared to serum. In controls (blue), CSF IgG1 was also less galactosylated but contained more afucosylated lgG1 ( $p_{\text {adj }}<0.01$ for all)

MS cases (reduced; bisecting GlcNAc and galactosylation; elevated in serum; sialylation; Additional file 5: Table S1).

Despite these absolute differences, IgG1 and IgG2 glycosylation was related. In serum, the proportions of bisected, galactosylated or sialylated IgG2 correlated with the proportion of the respective IgG1 glycoforms (MS serum; $\varrho=0.73$ to $0.89, p<0.00005, p_{\text {adj }}<0.0005$ for all glycosylation features; control serum; $\varrho=0.61$ to 0.93 , $p<0.005, p_{\text {adj }}<0.05$ for all glycofeatures).

In CSF, this correlation of respective IgG2 and IgG1 glycoforms was much weaker and only present as a trend for most glycosylation features, especially in the MS group ( $\varrho 0.45$ to 0.84 for all; $p_{\text {adj }}<0.05$ for galactosylation and sialylation in control CSF; only as a trend for bisecting GlcNAc in control CSF and all glycosylation features in MS CSF).

\section{Discussion}

This study shows four main results. First, IgG1 glycosylation differed between CSF and serum, even in the control group without intrathecal IgG synthesis. Second, in MS patients vs. controls, the IgG1 glycosylation pattern was altered in CSF, but not in serum. Third, alterations of glycosylation occurred especially shortly after a relapse. Fourth, glycosylation patterns in CSF from MS cases correlated with the degree of intrathecal IgG1 synthesis and CSF cell counts.

\section{Implications of CSF IgG1 glycosylation patterns for their effector functions}

IgG glycosylation regulates Fc effector functions. A proinflammatory pattern consists of elevated bisecting GlcNAc but reduced galactosylation [45], as we observed for IgG from CSF of MS patients compared to controls. The functional relevance of IgG Fc glycosylation patterns has been shown in animal models of systemic autoimmune diseases [10-14] and is consistent with observations in serum from humans with autoimmune diseases. The decreased IgG galactosylation in CSF in MS we describe here parallels a similar observation of reduced galactosylation of anti-citrullinated protein antibodies in synovial fluid in rheumatoid arthritis [46], as well as reduced serum IgG galactosylation in rheumatoid diseases [47]. There is little data on autoimmune diseases and IgG containing bisecting GlcNAc, but they were also elevated in the serum of LEMS patients [24]. In addition, reduced sialylation is thought to be a proinflammatory feature [8], which did not reach statistical significance in our cohort, possibly because of the overall low degree of sialylation. Taken together, these alterations we describe here in CSF IgG1 from MS patients (reduced galactosylation, increased bisecting GlcNAc) suggest that the CSF IgG1 in MS patients has enhanced IgG effector functions, resulting in a higher pro-inflammatory activity than that of controls.

For the decrease of afucosylation, as observed in CSF IgG1 from MS patients, the interpretation is more complex; afucosylation has initially been regarded as pro-inflammatory by enhancing ADCC via Fc $\gamma$ RIIIa [45]. A recent report confirmed this ADCC-enhancing effect of afucosylated IgG, but also reported an opposite effect, namely reduction of complement activation by afucosylation in the case of a therapeutic CD20 depleting antibody [22]. Thus, the decrease of afucosylation in the CSF of MS patients, compared to controls, may result in enhanced complement activation, but less ADCC. In fact, lesional complement activation was reported in the majority of RR-MS patients with early active lesions [6], and in an EAE model, autoantibody-mediated demyelination depended on complement activation but not on activatory Fc-receptors [48]. Nevertheless, Fc gamma receptors show elevated expression in MS lesions on microglia, albeit their exact role in MS is incompletely understood [49]. Therefore, the net outcome of the decrease of afucosylation in the MS group cannot be judged definitely, and we cannot exclude that it might also play a regulatory role for FcyRIIIa activation. In any case, the decrease of afucosylation in MS paralleled the findings in anti-citrullinated protein antibodies in rheumatoid arthritis [28].

Of note, MS-related changes in CSF IgG glycosylation where not only evident on the inter-group-level, but were linked to signs of inflammation also within the MS 


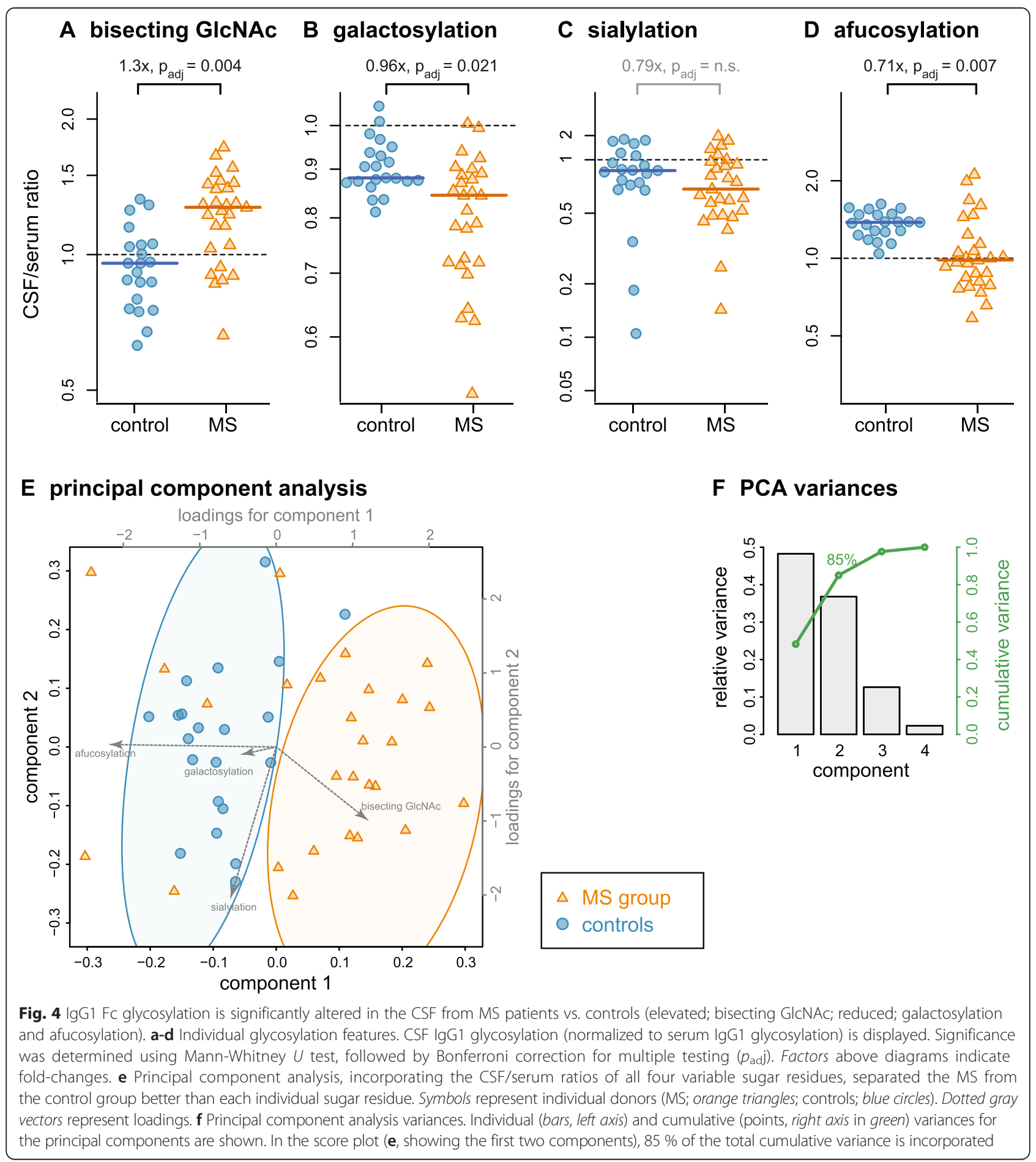

group, especially that the alteration in afucosylation (and as a trend also in galactosylation and bisecting GlcNAc) was more pronounced in those patients with stronger signs of intrathecal inflammation (higher CSF cell count and intrathecal IgG production).

When all four glycosylation features were combined by principal component analysis, separation of the groups was of similar discriminatory power as established markers such as CSF cell count or the intrathecal IgG fraction. However, there is no evidence that this inflammatory pattern of glycosylation is MS specific, but might rather be associated with the degree of inflammation. Using the glycopatterns and type of analysis we describe here, future studies can 


\section{A afucosylation}

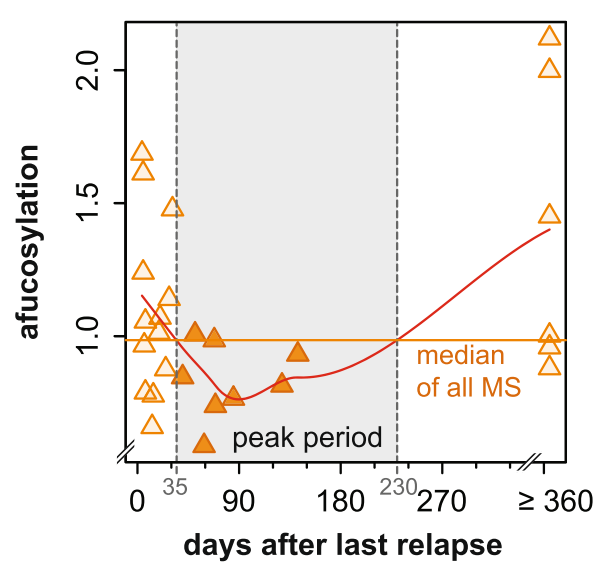

B bisecting GIcNAc

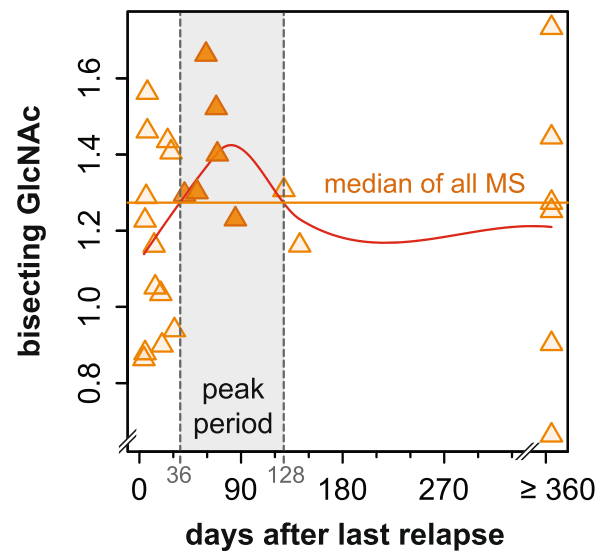

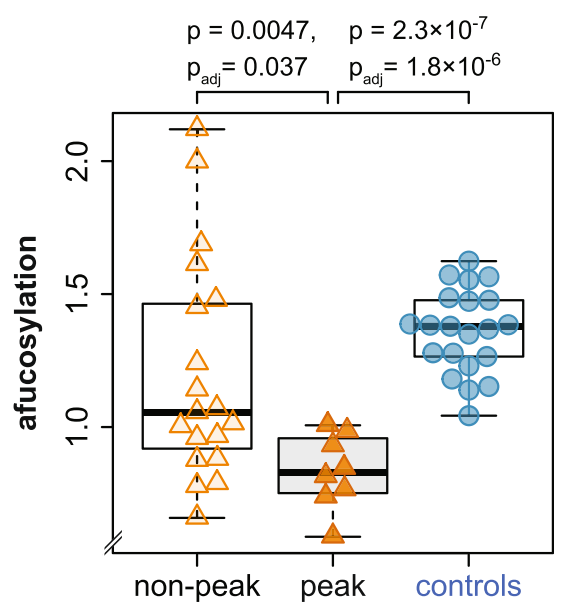

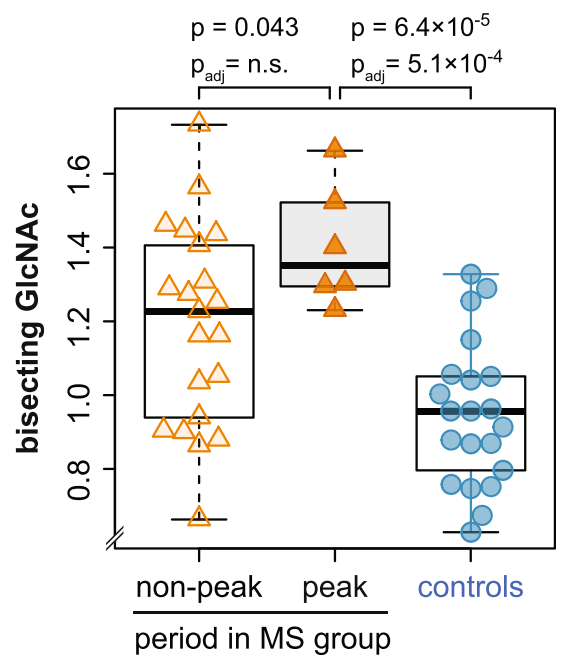

Fig. 5 Relationship between glycosylation and time since last relapse. Left part (scatterplots); afucosylation (a) and bisecting GlcNAc (b) in the CSF were plotted against the time since the last relapse. Samples from patients who did not have a relapse within 1 year of sampling were plotted at a cutoff of 1 year. Hypothesis-free regression was computed using the LOWESS method (red curve), demonstrating a peak of alterations 2-3 months after the last relapse. The period of the peak, defined by the crossings of the red LOWESS line with the median of all MS samples (horizontal line) was designated as peak period (gray area). Right part (boxplots): Samples from within this peak period were compared with samples outside of this period, and in addition also with control samples (one-sided $U$ test according to the directed hypothesis, followed by Bonferroni correction for the four lgG1 glycofeatures tested)

now apply these methods to address a number of obvious issues, such as the predictive value in addition to oligoclonal bands, comparison of autoimmune and infectious CNS diseases, alterations during aging, and effects of immunotherapy.

\section{Potential cause of altered IgG glycosylation}

We conclude from our data that it is the intrathecal production of IgG that results in an altered glycosylation pattern for the following reasons. First, MS-related glycosylation abnormalities were present in CSF, but not

Table 2 Summary of alterations in IgG1 glycosylation related to the compartment (CSF vs. serum, see also Fig. 3) and the disease (MS vs. control CSF, Fig. 4a-d, g)

\begin{tabular}{llll}
\hline & Control CSF vs. control serum & MS CSF vs. control CSF & Peak after relapse \\
\hline Afucosylation & $\uparrow$ & $\downarrow$ & $y$ \\
Bisecting GlcNAc & n.s. & $\uparrow$ & $y$ \\
Galactosylation & $\downarrow$ & $\downarrow$ & $n$ \\
\hline
\end{tabular}




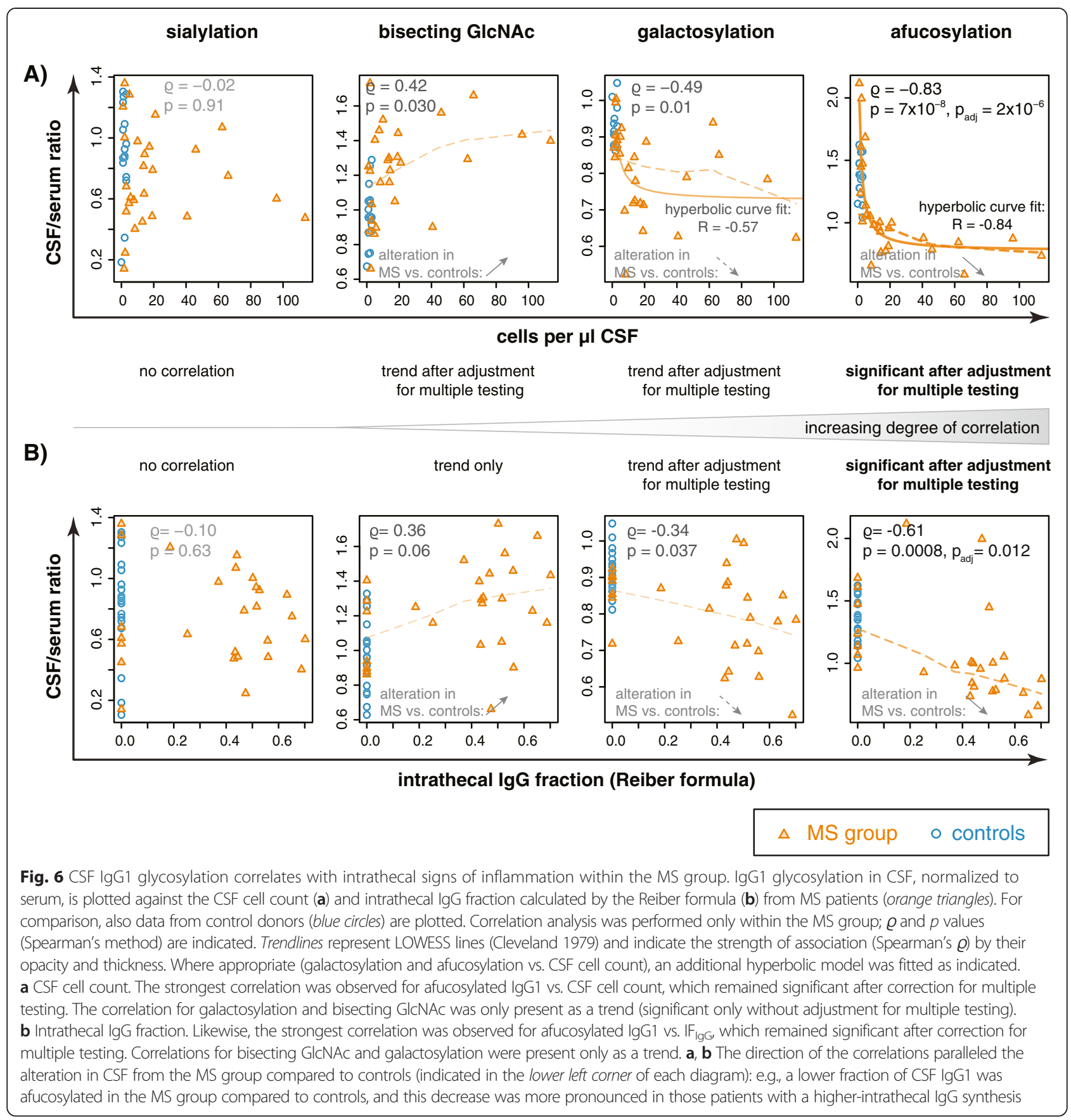

in blood. Second, the degree of these abnormalities (esp. afucosylation) in the CSF from MS patients correlated with the fraction of IgG that was actually produced intrathecally $\left(\mathrm{IF}_{\mathrm{IgG}}\right)$. Third, the strong correlation of CSF and serum IgG glycosylation in control donors was much weaker in the MS group, where intrathecally produced IgG occurs in addition to circulation-derived IgG. Fourth, the group differences between controls and MS patients were greater for IgG1 than for IgG2, and intrathecally produced $\operatorname{IgG}$ is mainly IgG1 $[50,51]$.
Previous cell culture experiments have provided evidence that the cytokine milieu and $\mathrm{pH}$ determine the glycopattern of secreted IgG $[52,53]$. Thus, the inflammatory milieu in the MS CNS is a likely cause for the pro-inflammatory glycofeatures of locally produced IgG that we observed in the CSF of patients with MS. This could constitute a positive feedback loop for CNS inflammation, reminiscent of a loop between dys-glycosylated myelin and inflammation [54].

Of note, IgG1 afucosylation and the presence of bisecting GlcNAc peaked 2-3 months after a clinical 
relapse. Even though the half-life of IgG1 is uncertain in the CSF (serum; 21 days), the kinetics were compatible with an inflammatory glycosylation pattern building up at the time of a relapse. It is possible that incorporating data on subclinical MRI activity and lesion localization would result in even better correlation of glycosylation patterns and disease features, but frequent MRI data were not available.

IgG glycosylation features were largely independent of each other within the same group and compartment, except in the case of sialylation and galactosylation, which highly correlated with each other. This seems plausible, given the subsequent addition of terminal sialic acid on top of galactose (Fig. 1). However, since sialylation is much lower than galactosylation, there would be enough space for independent degrees of sialylation and galactosylation. Therefore, the strong correlation of sialylation and galactosylation suggests that these two glycosyltransferases might be regulated in parallel, whereas regulation of the other MS-related glycosylation features may be mechanistically different.

\section{Even in the normal CSF, IgG glycosylation is distinct from serum}

An unexpected finding in this study was that IgG glycosylation in the CSF is distinct from serum even in the absence of inflammation and intrathecal IgG production. Although these differences were small, they were significant also after adjustment for multiple testing. Potential explanations include that (1) IgG transport into or (2) half-life within the CSF compartment depends on glycosylation, or that (3) IgG glycosylation is modified in the CSF or serum.

Galactosylation was reduced in control CSF and could thus favor IgG effector functions also in healthy subjects, but it is likely that a further reduction of galactosylation, as observed in MS (Fig. 4), as well as the presence of complement and cells that mediate ADCC, which are present in MS lesions, are necessary to actually unleash IgG effector functions. Of note, the pattern of differences between control CSF and serum, and those between MS and control CSF, were not identical; in particular, afucosylation was increased in control CSF vs. serum but decreased in MS vs. control CSF.

\section{Limitations of this study}

Since clinical samples were collected at different sites, we cannot completely rule out any site bias. However, in order to minimize any pre-analytical issues, samples were collected following the same protocol, centrifuged and frozen immediately, shipped on dry ice, and analyzed by mass spectrometry altogether as detailed in the Methods section. Analyzing the CSF/serum ratios of the MS vs. the control group separately for site 1 and site 2, we observed similar changes also within the samples of site 1 or 2 for the glycofeatures with significant regulation, arguing against a major site bias.

\section{Conclusions}

The CNS compartment as well as the inflammatory milieu in MS affect IgG1 Fc glycosylation. In MS, the altered CSF IgG1 glycosylation pattern has proinflammatory features and is linked to intrathecal IgG synthesis (see also Fig. 1 and Table 2 for summarizing depiction; Fig. 6). We suggest that the inflammatory intrathecal milieu in MS might cause the described proinflammatory IgG glycosylation pattern, which in turn might further support pro-inflammatory IgG effector mechanisms, possibly constituting a vicious circle that helps to perpetuate the inflammatory process in MS.

\section{Additional files}

\begin{abstract}
Additional file 1: Figure S1. Representative mass spectrometric lgG1 and IgG2 Fc glycosylation data from an MS patient. Tryptic Fc glycopeptides of $\operatorname{lgG} 1$ and $\lg G 2$ isolated from (A) serum and (B) cerebrospinal fluid (CSF) from a MS patient were analyzed by MALDIFTICR-MS. IgG1 (continued arrow) and lgG2 (striated arrow) glycopeptide signals with identical glycan portions were registered as peak pairs due to a 32-Da mass difference of the peptide moieties. The inset shows the signals obtained for two sialylated glycopeptide species. pep peptide moiety. Symbols and colors are drawn according to the Consortium for Functional Glycomics [55]. (PDF $236 \mathrm{~kb}$ )

Additional file 2: Figure S2. CSF vs. serum lgG2 glycosylation. Afucosylation could only be assessed for $\lg \mathrm{G} 1$, but not for $\lg \mathrm{G} 2$, as several fucosylated lgG2 glycoforms could not be determined due to overlay with lgG4 glycan structures. Individual data points are horizontally jittered to avoid obscuring them from each other. Lines indicate corresponding CSF/serum pairs but do not necessarily end directly at the horizontally jittered data points to preserve angles of the connecting lines. Significance was determined using Wilcoxon-signed rank test for paired samples, followed by Bonferroni correction for multiple testing $\left(p_{\text {adj }}\right)$. Factors above diagrams indicate fold-changes (medians of paired CSF/serum ratios). (PDF $326 \mathrm{~kb}$ )
\end{abstract}

Additional file 3: Figure S3. IgG2 glycosylation in CSF and serum from MS patients vs. controls. CSF IgG2 glycosylation (normalized to serum IgG2 glycosylation) is displayed. Significance was determined using Mann-Whitney $U$ test, followed by Bonferroni correction for multiple testing $\left(p_{\text {adj }}\right)$. Factors above diagrams indicate fold-changes. IgG2 afucosylation could not be assessed because of overlay with lgG4 glycan structures. (PDF $169 \mathrm{~kb}$ )

Additional file 4: Figure S4. Terminal lgG1 sialylation and galactosylation correlate with each other (A), whereas other combinations of glycosylation features within the same group and compartment do not (B). $\varrho$ and $p$ values (Spearman's method, adjusted for multiple testing) are given for each diagram. Trendlines represent LOWESS lines (Cleveland 1979) and indicate the strength of association by their opacity and thickness. (PDF 254 kb)

Additional file 5: Table S1. IgG2 vs. IgG1 glycosylation. Displayed are ratios (lgG2/lgG1) for each glycofeature within the same group and compartment. $p_{\text {adj }}$ denotes the $p$ value after Bonferroni correction. IgG2 afucosylation could not be assessed because of overlay with lgG4 glycan structures. (DOC $32 \mathrm{~kb}$ )

\section{Abbreviations}

CSF: cerebrospinal fluid; GlcNAc: N-acetylglucosamine; lgG: immunoglobulin G; MS: multiple sclerosis; Fc: fragment crystallizable; FcyR: Fc gamma receptor; $\mathrm{CH}_{2}$ : constant heavy domain 2. 


\section{Competing interests}

MW and MHJS received research grant support from Hoffmann La Roche. LAM reports no competing interests. TK received travel expenses and personal compensations (speaker honoraria) from Bayer Healthcare, Teva Pharma, Merck Serono, Novartis, Genzyem/Sanofi-Aventis, and Biogen as well as grant support from Bayer Schering AG and Novartis. TD serves on scientific advisory boards for Novartis Pharmaceuticals, Merck Serono, Biogen Idec, Genzyme, GeNeuro, Mitsubishi Pharma, Teva Pharmaceuticals and Bayer Schering Pharma; has received funding for travel and/or speaker honoraria from Biogen Idec, Genzyme, Novartis, Merck Serono and Bayer Schering Pharma; and receives research support from Biogen Idec, Novartis Pharma, the European Union, the Swiss National Foundation and the Swiss MS Society. MK reports no competing interests. TO received unrestricted MS research grants from the following companies: Biogen, Novartis, Genzyme, Almirall. The same companies have given compensation for advisory boards/ and or lectures. $\mathrm{RH}$ received personal compensations for activities such as advisory board, and/or consultancy fees from Teva, Genzyme, Sanofi, Bayer Schering, Merck Serono, Biogen Idec, Novartis, Behring CSL, Morphosys, Actelion, and research grant support from Teva, Bayer Schering, Serono, Biogen Idec, Novartis and Genzyme-Sanofi. EM received grant support by Novartis and advisory board compensations from Roche. MKr received grant support, traveling expenses and scientific advisory board honoraria from Novartis, the Novartis foundation and Genzyme.

\section{Authors' contributions}

MW, MHJS, and LAM performed experiments. MW, MHJS, LAM, and MKr analyzed data. TK, TD, MK and TO contributed important and valuable patient samples and patient data. MKr, MW, and EM drafted the manuscript. All authors worked on the final text version. MKr designed the study, and $\mathrm{MW}, \mathrm{RH}$, and EM supervised the study. All authors read and approved the final manuscript.

\section{Acknowledgements}

The authors wish to thank Evelyne Steenvoorden, Heike Rübsamen, Sabine Pitter, Angelika Bamberger, and Anton Dischner for expert technical assistance, Anne Waschbisch for help with collecting patient samples, Kerstin Berer and Naoto Kawakami for helpful comments on the manuscript, and all healthy volunteers, patients, nurses, and other physicians for serum and CSF samples.

\section{Funding}

This study was supported by the Deutsche Forschungsgemeinschaft (SFB TR128, to EM and RH), BMBF (Krankheitsbezogenes Kompetenznetz Multiple Sklerose, to EM and $\mathrm{RH}$ ), the European Commission (FP7 project HighGlycan; contract \#278535, to MW), the Gemeinnützige Hertie Foundation, and the Verein zur Therapieforschung für Multiple Sklerose-Kranke (to EM, RH). TO received academic research grants from the Swedish Research council, Swedish Brain foundation, AFA foundation, Knut and Alice Wallenberg Foundation, and Margareta Af Ugglas foundation. The funding organizations had no influence on the design, conduct, and analysis of this study.

\section{Author details}

${ }^{1}$ Center for Proteomics and Metabolomics, Leiden University Medical Center, Leiden, The Netherlands. ${ }^{2}$ Department of Molecular Cell Biology and Immunology, VU University Medical Center, Amsterdam, The Netherlands. ${ }^{3}$ Division of BioAnalytical Chemistry, VU University Amsterdam, Amsterdam, The Netherlands. ${ }^{4}$ Institute of Clinical Neuroimmunology, Biomedical Center (BMC) and University Hospital, Campus Martinsried-Grosshadern, LMU Munich, Munich, Germany. ${ }^{5}$ Departments of Neurology and Biomedicine, University Hospital, Basel, Switzerland. ${ }^{6}$ Department of Clinical Neuroscience, Neuroimmunology Unit, Karolinska University Hospital, Stockholm, Sweden. ${ }^{7}$ Munich Cluster of Systems Neurology (SyNergy), Munich, Germany. ${ }^{8}$ Department of Neurology and Stroke, and Hertie Institute for Clinical Brain Research, University of Tübingen, Tübingen, Germany.

Received: 16 June 2015 Accepted: 2 December 2015

Published online: 18 December 2015

\section{References}

1. Stangel M, Fredrikson S, Meinl E, Petzold A, Stuve O, Tumani H. The utility of cerebrospinal fluid analysis in patients with multiple sclerosis. Nat Rev Neurol. 2013;9(5):267-76. doi:10.1038/nrneurol.2013.41.

2. von Büdingen HC, Bar-Or A, Zamvil SS. B cells in multiple sclerosis: connecting the dots. Curr Opin Immunol. 2011;23(6):713-20. http://dx.doi. org/10.1016/j.coi.2011.09.003.

3. Antel J, Bar-Or A. Roles of immunoglobulins and B cells in multiple sclerosis: from pathogenesis to treatment. J Neuroimmunol. 2006;180(1-2):3-8.

4. Elliott $C$, Lindner M, Arthur A, Brennan K, Jarius S, Hussey J, et al. Functional identification of pathogenic autoantibody responses in patients with multiple sclerosis. Brain. 2012;135(Pt 6):1819-33. doi:10.1093/brain/aws105.

5. Storch MK, Piddlesden S, Haltia M, livanainen M, Morgan P, Lassmann $H$. Multiple sclerosis: in situ evidence for antibody- and complement-mediated demyelination. Ann Neurol. 1998;43(4):465-71.

6. Lucchinetti C, Bruck W, Parisi J, Scheithauer B, Rodriguez M, Lassmann H. Heterogeneity of multiple sclerosis lesions: implications for the pathogenesis of demyelination. Ann Neurol. 2000;47(6):707-17.

7. Krumbholz M, Derfuss T, Hohlfeld R, Meinl E. B cells and antibodies in multiple sclerosis pathogenesis and therapy. Nat Rev Neurol. 2012;8(11):613-23.

8. Lux A, Nimmerjahn F. Impact of differential glycosylation on IgG activity crossroads between innate and adaptive immunity III. In: Pulendran B, Katsikis PD, Schoenberger SP, editors. Advances in Experimental Medicine and Biology. New York: Springer; 2012. p. 113-24.

9. Huhn C, Selman MHJ, Ruhaak LR, Deelder AM, Wuhrer M. IgG glycosylation analysis. Proteomics. 2009;9(4):882-913. doi:10.1002/pmic.200800715.

10. Rademacher TW, Williams P, Dwek RA. Agalactosyl glycoforms of lgG autoantibodies are pathogenic. Proc Natl Acad Sci. 1994;91(13):6123-7.

11. Karsten CM, Pandey MK, Figge J, Kilchenstein R, Taylor PR, Rosas M, et al. Anti-inflammatory activity of IgG1 mediated by Fc galactosylation and association of FcgammaRIIB and dectin-1. Nat Med. 2012;18(9):1401-6. doi:10.1038/nm.2862.

12. Schwab I, Nimmerjahn F. Intravenous immunoglobulin therapy: how does IgG modulate the immune system? Nat Rev Immunol. 2013;13(3):176-89.

13. Nandakumar KS, Collin M, Olsen A, Nimmerjahn F, Blom AM, Ravetch JV, et al. Endoglycosidase treatment abrogates lgG arthritogenicity: importance of IgG glycosylation in arthritis. Eur J Immunol. 2007;37(10):2973-82.

14. Albert H, Collin M, Dudziak D, Ravetch JV, Nimmerjahn F. In vivo enzymatic modulation of lgG glycosylation inhibits autoimmune disease in an lgG subclass-dependent manner. Proc Natl Acad Sci. 2008;105(39):15005-9.

15. Kaneko Y, Nimmerjahn F, Ravetch JV. Anti-inflammatory activity of immunoglobulin $\mathrm{G}$ resulting from Fc sialylation. Science. 2006:313(5787):670-3.

16. Anthony RM, Nimmerjahn F, Ashline DJ, Reinhold VN, Paulson JC, Ravetch JV. Recapitulation of IVIG anti-inflammatory activity with a recombinant IgG FC. Science. 2008;320(5874):373-6.

17. von Gunten S, Shoenfeld Y, Blank M, Branch DR, Vassilev T, Kasermann F, et al. IVIG pluripotency and the concept of Fc-sialylation: challenges to the scientist. Nat Rev Immunol. 2014;14(5):349. doi:10.1038/nri3401-c1.

18. Schwab I, Lux A, Nimmerjahn F. Reply to [mdash] IVIG pluripotency and the concept of Fc-sialylation: challenges to the scientist. Nat Rev Immunol. 2014;14(5):349. doi:10.1038/nri3401-c2.

19. Ito K, Furukawa J-i, Yamada K, Tran NL, Shinohara Y, Izui S. Lack of galactosylation enhances the pathogenic activity of $\lg$ G1 but not lgG2a anti-erythrocyte autoantibodies. J Immunol. 2014;192(2):581-8. doi:10.4049/ jimmunol.1302488.

20. Lifely MR, Hale C, Boyce S, Keen MJ, Phillips J. Glycosylation and biological activity of CAMPATH-1H expressed in different cell lines and grown under different culture conditions. Glycobiology. 1995;5(8):813-22.

21. Umana P, Jean-Mairet J, Moudry R, Amstutz H, Bailey JE. Engineered glycoforms of an antineuroblastoma lgG1 with optimized antibodydependent cellular cytotoxic activity. Nat Biotech. 1999;17(2):176-80.

22. Gasdaska JR, Sherwood S, Regan JT, Dickey LF. An afucosylated anti-CD20 monoclonal antibody with greater antibody-dependent cellular cytotoxicity and B-cell depletion and lower complement-dependent cytotoxicity than rituximab. Mol Immunol. 2012;50(3):134-41.

23. Parekh RB, Dwek RA, Sutton BJ, Fernandes DL, Leung A, Stanworth D, et al. Association of rheumatoid arthritis and primary osteoarthritis with changes in the glycosylation pattern of total serum IgG. Nature. 1985;316(6027):452-7. 
24. Selman MH, Niks EH, Titulaer MJ, Verschuuren JJ, Wuhrer M, Deelder AM. IgG fC $\mathrm{N}$-glycosylation changes in Lambert-Eaton myasthenic syndrome and myasthenia gravis. J Proteome Res. 2011;10(1):143-52. doi:10.1021/pr1004373.

25. Fokkink W-JR, Selman MHJ, Dortland JR, Durmuş B, Kuitwaard K, Huizinga $\mathrm{R}$, et al. IgG FC N-Glycosylation in Guillain-Barré syndrome treated with immunoglobulins. J Proteome Res. 2014;13(3):1722-30. doi:10.1021/pr401213z.

26. Albrecht S, Unwin L, Muniyappa M, Rudd PM. Glycosylation as a marker for inflammatory arthritis. Cancer Biomark. 2014;14(1):17-28. doi:10.3233/cbm-130373.

27. Ercan A, Barnes MG, Hazen M, Tory H, Henderson L, Dedeoglu F, et al. Multiple juvenile idiopathic arthritis subtypes demonstrate proinflammatory IgG glycosylation. Arthritis Rheum. 2012;64(9):3025-33. doi:10.1002/art.34507.

28. Rombouts Y, Ewing E, van de Stadt LA, Selman MHJ, Trouw LA, Deelder AM, et al. Anti-citrullinated protein antibodies acquire a pro-inflammatory FC glycosylation phenotype prior to the onset of rheumatoid arthritis. Ann Rheum Dis. 2013. doi:10.1136/annrheumdis-2013-203565.

29. Bradl M, Misu T, Takahashi T, Watanabe M, Mader S, Reindl M, et al. Neuromyelitis optica: pathogenicity of patient immunoglobulin in vivo. Ann Neurol. 2009;66(5):630-43.

30. Bennett JL, Lam C, Kalluri SR, Saikali P, Bautista K, Dupree C, et al. Intrathecal pathogenic anti-aquaporin-4 antibodies in early neuromyelitis optica. Ann Neurol. 2009;66(5):617-29.

31. Tradtrantip L, Ratelade J, Zhang H, Verkman AS. Enzymatic deglycosylation converts pathogenic neuromyelitis optica anti-aquaporin-4 immunoglobulin $\mathrm{G}$ into therapeutic antibody. Ann Neurol. 2013;73(1):77-85. doi:10.1002/ana.23741.

32. Collin $M$, Olsen A. Effect of SpeB and EndoS from streptococcus pyogenes on human immunoglobulins. Infect Immun. 2001;69(11):7187-9.

33. Benkhoucha M, Molnarfi N, Santiago-Raber ML, Weber MS, Merkler D Collin $\mathrm{M}$, et al. IgG glycan hydrolysis by EndoS inhibits experimental autoimmune encephalomyelitis. J Neuroinflammation. 2012;9:209. doi:10.1186/1742-2094-9-209.

34. van Timmeren MM, van der Veen BS, Stegeman CA, Petersen AH, Hellmark T, Collin $\mathrm{M}$, et al. IgG glycan hydrolysis attenuates ANCA-mediated glomerulonephritis. J Am Soc Nephrol. 2010;21(7):1103-14.

35. Yang R, Otten MA, Hellmark T, Collin M, Björck L, Zhao MH, et al. Successful treatment of experimental glomerulonephritis with IdeS and EndoS, IgG-degrading streptococcal enzymes. Nephrol Dial Transplant. 2010;25(8):2479-86.

36. Song T, Ozcan S, Becker A, Lebrilla CB. In-depth method for the characterization of glycosylation in manufactured recombinant monoclonal antibody drugs. Anal Chem. 2014;86(12):5661-6. doi:10.1021/ac501102t.

37. Jefferis R. Glycosylation as a strategy to improve antibody-based therapeutics. Nat Rev Drug Discov. 2009;8(3):226-34.

38. Reiber H, Peter JB. Cerebrospinal fluid analysis: disease-related data patterns and evaluation programs. J Neurol Sci. 2001;184(2):101-22.

39. Shikata K, Yasuda T, Takeuchi F, Konishi T, Nakata M, Mizuochi T. Structural changes in the oligosaccharide moiety of human $\lg G$ with aging. Glycoconj J. 1998;15(7):683-9.

40. Pucic M, Knezevic A, Vidic J, Adamczyk B, Novokmet M, Polasek O, et al. High throughput isolation and glycosylation analysis of lgG - variability and heritability of the lgG glycome in three isolated human populations. Mol Cell Proteomics. 2011;10(10):M111.010090.

41. Selman MHJ, McDonnell LA, Palmblad M, Ruhaak LR, Deelder AM, Wuhrer M. Immunoglobulin G glycopeptide profiling by matrix-assisted laser desorption ionization fourier transform ion cyclotron resonance mass spectrometry. Anal Chem. 2010;82(3):1073-81. doi:10.1021/ac9024413.

42. R Core Team. R: A language and environment for statistical computing. 301st ed. Vienna, Austria: R Foundation for Statistical Computing; 2013.

43. Benjamini $Y$, Hochberg $Y$. Controlling the false discovery rate-a practical and powerful approach to multiple testing. J R Stat Soc Ser B Methodol. 1995;57(1):289-300.

44. Cleveland WS. Robust locally weighted regression and smoothing scatterplots. J Am Stat Assoc. 1979;74(368):829-36.

45. Lux A, Yu X, Scanlan CN, Nimmerjahn F. Impact of immune complex size and glycosylation on IgG binding to human FcyRs. J Immunol. 2013;190(8):4315-23. doi:10.4049/jimmunol.1200501.

46. Scherer HU, van der Woude D, loan-Facsinay A, el Bannoudi H, Trouw LA, Wang J, et al. Glycan profiling of anti-citrullinated protein antibodies isolated from human serum and synovial fluid. Arthritis Rheum. 2010;62(6):1620-9.
47. Collin M, Ehlers M. The carbohydrate switch between pathogenic and immunosuppressive antigen-specific antibodies. Exp Dermatol. 2013;22(8):511-4. doi:10.1111/exd.12171.

48. Urich E, Gutcher I, Prinz M, Becher B. Autoantibody-mediated demyelination depends on complement activation but not activatory Fc-receptors. Proc Natl Acad Sci. 2006;103(49):18697-702. doi:10.1073/pnas.0607283103.

49. Torkildsen O, Vedeler CA, Nyland HI, Myhr KM. FcgammaR and multiple sclerosis: an overview. Acta Neurol Scand Suppl. 2006;183:61-3.

50. Eickhoff K, Kaschka W, Skvaril F, Theilkaes L, Heipertz R. Determination of IgG subgroups in cerebrospinal fluid of multiple sclerosis patients and others. Acta Neurol Scand. 1979;60(5):277-82.

51. Losy J, Michalowska-Wender G, Wender M. IgG1-lgG4 subclasses in the cerebrospinal fluid and blood serum and their synthesis in the central nervous system in multiple sclerosis. Neurol Neurochir Pol. 1992;26(3):297-303.

52. Wang J, Balog CIA, Stavenhagen K, Koeleman CAM, Scherer HU, Selman MHJ, et al. Fc-glycosylation of IgG1 is modulated by B-cell stimuli. Mol Cell Proteomics. 2011;10(5):M110.004655.

53. Müthing J, Kemminer SE, Conradt HS, Sagi D, Nimtz M, Kärst U, et al. Effects of buffering conditions and culture $\mathrm{pH}$ on production rates and glycosylation of clinical phase I anti-melanoma mouse lgG3 monoclonal antibody R24. Biotechnol Bioeng. 2003;83(3):321-34.

54. García-Vallejo JJ, Ilarregui JM, Kalay H, Chamorro S, Koning N, Unger WW, et al. CNS myelin induces regulatory functions of DC-SIGN-expressing, antigen-presenting cells via cognate interaction with MOG. J Exp Med. 2014;211(7):1465-83. doi:10.1084/jem.20122192.

55. Functional glycomics gateway. Symbol and text nomenclature for representation of glycan structure. http://www.functionalglycomics.org/ static/consortium/Nomenclature.shtml. Accessed 05/28/2014.

\section{Submit your next manuscript to BioMed Central and we will help you at every step:}

- We accept pre-submission inquiries

- Our selector tool helps you to find the most relevant journal

- We provide round the clock customer support

- Convenient online submission

- Thorough peer review

- Inclusion in PubMed and all major indexing services

- Maximum visibility for your research

Submit your manuscript at www.biomedcentral.com/submit 